\title{
Designer Nanoparticle-Liposome Hybrid Capsules for Drug Delivery
}

\author{
Joseph A Zasadzinski, Jeong Eun Shin, Natalie Forbes and Maria Ogunyankin.
}

Chemical Engineering and Materials Science, University of Minnesota, Minneapolis, Minnesota 55455.

Unilamellar liposomes can become the building blocks of increasingly complex nanostructures that cn optimize drug retention, targeting to specific sites, and controlled release rates. Tethering or encapsulating near infrared light adsorbing gold nanoparticles [1,2] provides new targeting and release mechanisms with physiologically friendly near infrared light, while retaining the advantages of liposome biocompatibility and biodistribution. Multiple levels of encapsulation within multiple lipid bilayers [3, 4] can prolong drug retention by orders of magnitude over unilamellar liposomes. It is often difficult to maintain drug levels within the concentration range necessary to avoid toxicity while maintaining efficacy using systemic drug delivery. This therapeutic window can be expanded by altering drug biodistribution by using nanoscale delivery systems to maximize concentration at the disease site while decreasing toxicity by lowering drug concentrations elsewhere in the body. Nanoscale delivery vehicles can take advantage of the enhanced permeability and retention (EPR) effect, which is a passive targeting mechanism that exploits the rapid angiogenesis (formation of new blood vessels from existing ones) of tumor and inflammation sites. Whereas free drugs diffuse non-specifically, a nanocarrier can escape preferentially through the leaky vessels surrounding a tumor, which is the first step of the EPR effect. The ineffective lymphatic drainage in tumors helps retain liposomes that do extravasate and allows the liposomes to accumulate in the neighborhood of the tumor. The threshold size for extravasation into tumors is from $400-500 \mathrm{~nm}$, but smaller nanoparticles with diameters less than $200 \mathrm{~nm}$ are believed to be more effective. The minimum size is about $5 \mathrm{~nm}$, which is the kidney filtration cut off size.

Encapsulating doxorubicin within PEGylated liposomes significantly reduced cardiotoxicity and increased the half-life in the systemic circulation. However, efficacy did not improve as expected from the increased liposomal doxorubicin accumulation at the tumor site [5] because of overly slow drug release. Liposomal cisplatin extended systemic half-life to $40-55 \mathrm{hrs}$ [6] from the $15-20 \mathrm{~min}$ clearance of free cisplatin. However, cisplatin was not released at a therapeutic rate from the liposomes, even though 5- to 10-fold greater liposome encapsulated cisplatin accumulated in the tumors. This highlights the conflict between "retain" and "release" - factors that enhance drug retention often work against an optimal rate of drug release. Hybrid gold nanoparticle-liposome carriers provide a noninvasive method to initiate, control, and even stop drug release via membrane-localized temperature control. Plasmonic hollow gold nanoshells (HGN) are tethered (Fig. 1A) to or encapsulated within (Fig. 1B) liposomes via thiol-PEG-lipids to create a hybrid nanocarrier. The liposome-HGN are irradiated with picosecond pulses or continuous wave near infra-red (NIR) light. Picosecond pulses induce a rapid temperature rise in the HGN, leading to formation of vapor nanobubbles, similar to the cavitation bubbles caused by sonication, rupturing the liposomes and causing near instantaneous contents release. Continuous wave NIR irradiation leads to much smaller temperature increases near the liposome membrane that can induce permeability transitions in the liposome membrane, leading to contents release over minutes. The great advantage of using NIR light to induce release is that tissue, blood, etc. are relatively transparent to $650-950 \mathrm{~nm}$ wavelength light, allowing NIR transmission in soft tissues at depths up to several $\mathrm{cm}$. Only complexes irradiated by the laser release drug, providing a targeting mechanism. We find that rapid doxorubicin release at the tumor site can reduce the necessary doxorubicin dosage by a factor of 10 or more. 
While liposomal doxorubicin has been the most successful of all nanoscale drug carriers, many drugs, such as the antibiotic ciprofloxacin and the chemotherapy agents vinorelbine, vinblastine and vincristine, leak out too rapidly from liposomes, which severely limits their efficacy. While coating the exterior of liposomes with polyethylene glycol (PEG) extends in vivo circulation times to 24 - 48 hours, PEG-lipids actually increase liposome permeability. To address premature release, we have made multicompartment structure based on liposomes within liposomes or "vesosomes" (Fig. 1C) [3]. Eukaryotes developed this nested bilayer structure as an alternative to optimizing the chemistry and physics of a single bilayer; each compartment has its own distinct bilayer membrane which separates different functions while protecting the internal contents from the environment [3, 4]. Having two or more bilayers between the environment and the drug contents can make a significant difference in drug retention in physiological environments. The additional bilayer barrier effectively eliminates drug release caused by exposure to phospholipase A2 (PLA2). Contents release from unilamellar liposomes after exposure to PLA2 was complete within 4- 8 hours. No release beyond background was induced by PLA2 from vesosomes over 24 hours. This indicates that the PLA2 cannot traverse the exterior bilayer of the vesosome to degrade the internal vesicles during the time of the experiment. The vesosome structure extended the half-life for release from vesosomes in serum to more than 50 hours (from about 2 hours for unilamellar liposomes), and was a delay in release for $\sim 10$ hours before any significant contents release occurred. Ciprofloxacin release could be extended from 10 minutes to 10 hours in serum.

\section{References}

[1] G. Wu, et al., J Am. Chem. Soc. 130,(2008), p. 8175.

[2] N. Forbes, et al., Particles and Particle Systems Characterization 31,(2014), p. 1158.

[3] C. Boyer and J. A. Zasadzinski, ACS Nano 1,(2007), p.176.

[4] B. Wong, et al., Adv. Materials 23,(2011), p. 2320.

[5] M. E. R. O'Brien, et al., Ann. Oncol. 15,(2004), p. 440.

[6] W. C. Zamboni, et al., Cancer Chemother. Pharmacol. 53,(2004), p. 329.

[7] This work was supported by National Institutes of Health (NIH) grant R01 EB012637.
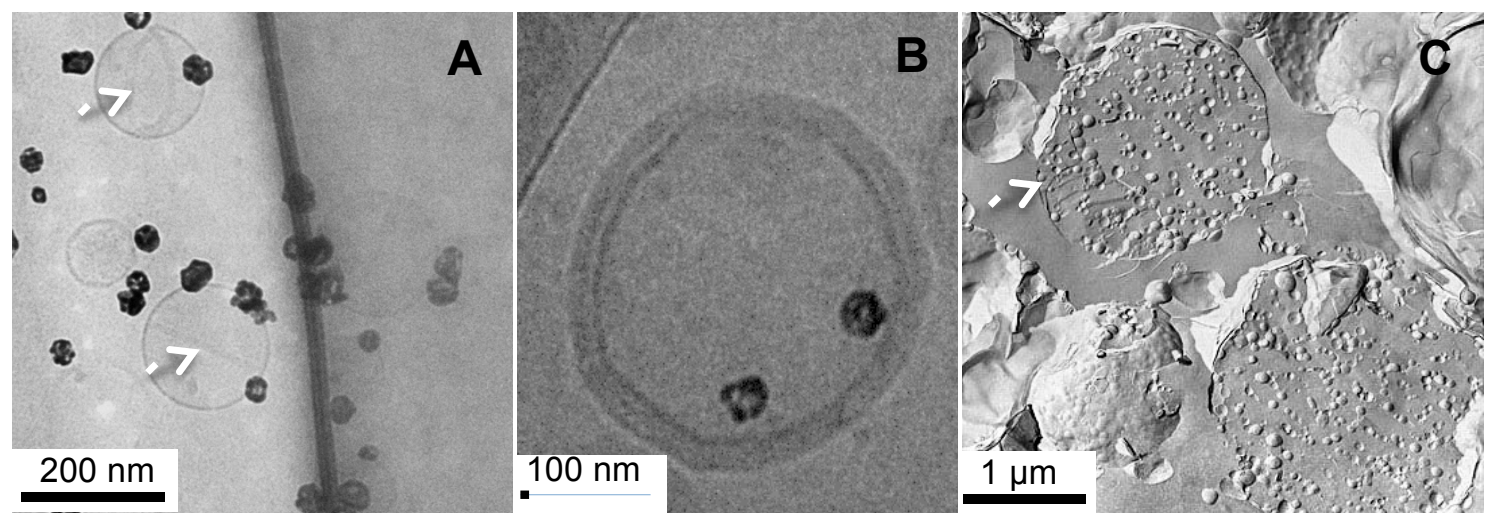

Figure 1. A) Cryo-TEM image of liposomes with tethered hollow gold nanoshells. The arrows point to encapsulated doxorubicin precipitates. B) HGN encapsulated within liposomes. Cryo-TEM is necessary to see within the liposome membranes. C) Freeze-fracture image of vesosomes. Arrow points to fractured exterior membrane. The interior vesicles are $\sim 50 \mathrm{~nm}$ in diameter. This multiple membrane structure can slow contents release by two orders of magnitude. 